\title{
Amplification of Cord Blood-Derived Cytotoxic T Lymphocytes using HL-60 cell Derived Exosomes
}

\section{Type}

Research paper

\section{Keywords}

dendritic cells, cord blood, adoptive immunotherapy, Exosome, cytotoxic T lymphocytes

\begin{abstract}
Introduction

Dendritic cell (DCs) based cytotoxic T lymphocytes (CTLs) are commonly used in immunotherapy due to their specificity. The selection of appropriate cell origin and tumor antigen is the key point. The objective was to culture DCs and CTLs simultaneously from cord blood, and deliver antigen information using tumor derived exosomes.

Material and methods

Exosomes were collected from the human promyelocytic leukemia cell line HL-60 using ultracentrifugation. Prepared DCs from adherent cord blood mononuclear cells (MNCs) using SCF, GM-CSF, and IN-4. TNF- $\alpha$ and microRNA removed tumor-exosome were used to induce DCs maturation. DCs matured in the presence of $\mathrm{HL}-60$ cell membrane protein extract or no antigen were set as control. CTLs was cultured from non-adherent MNCs by adding IFN- $\gamma$, IL-15, SCF, FLT-3L, anti-CD3, anti-CD28 and IL-2. The CTLs were analyzed by flow cytometry, cytotoxicity experiments and ELISA.
\end{abstract}

Results

DCs can be obtained from cord blood and express costimulatory molecules. After 15 days, the total number of the cells expanded 26.3 times, and more than $82 \%$ of the cells expressed CD3+CD8+ in the most amplified HL-60-Ex-DCs-CTL group. These CD3+CD8+ T cells generated by HL-60-ExDCs displayed specific cytotoxicity towards HL-60 and low lethality towards unrelated BALL-1 cells. ELISA results showed that the expressions of TNF- $\alpha$ and IFN-y in HL-60-Ex-DCs or HL-60mPr-DCs activated CTLs were upregulated compared with the control group.

\section{Conclusions}

Cord blood CTLs generated by HL-60 derived exosome activated DCs displayed specific cytotoxicity towards HL-60 promyelocytic leukemia cells. Therefore cord blood and tumor derived exosomes provided a good source for adoptive immunotherapy. 


\section{Amplification of Cord Blood-Derived Cytotoxic T Lymphocytes using}

\section{HL-60 cell Derived Exosomes}

Running title: Exosome actived DC amplify CTL

Keywords: cytotoxic T lymphocytes, dendritic cells, adoptive immunotherapy, exosome

\section{Abstract:}

Introduction: Dendritic cell (DCs) based cytotoxic T lymphocytes (CTLs) are commonly used in immunotherapy due to their specificity. The selection of appropriate cell origin and tumor antigen is the key point. The objective was to culture DCs and CTLs simultaneously from cord blood, and deliver antigen information using tumor derived exosomes.

Material and methods: Exosomes were collected from the human promyelocytic leukemia cell line HL-60 using ultracentrifugation. Prepared DCs from adherent cord blood mononuclear cells (MNCs) using SCF, GM-CSF, and IN-4. TNF- $\alpha$ and microRNA removed tumor-exosome were used to induce DCs maturation. DCs matured in the presence of HL-60 cell membrane protein extract or no antigen were set as control. CTLs was cultured from non-adherent MNCs by adding IFN- $\gamma$, IL-15, SCF, FLT-3L, anti-CD3, anti-CD28 and IL-2. The CTLs were analyzed by flow cytometry, cytotoxicity experiments and ELISA.

Results: DCs can be obtained from cord blood and express costimulatory molecules. After 15 days, the total number of the cells expanded 26.3 times, and more than $82 \%$ of the cells expressed $\mathrm{CD}^{+} \mathrm{CD}^{+}$in the most amplified HL-60-Ex-DCs-CTL group. These $\mathrm{CD} 3^{+} \mathrm{CD} 8^{+} \mathrm{T}$ cells generated by HL-60-Ex-DCs displayed specific cytotoxicity towards HL-60 and low lethality towards unrelated BALL-1 cells. ELISA results showed that the expressions of TNF- $\alpha$ and IFN- $\gamma$ in HL-60-Ex-DCs or HL-60mPr-DCs activated CTLs were upregulated compared with the control group.

Conclusion: Cord blood CTLs generated by HL-60 derived exosome activated DCs displayed specific cytotoxicity towards HL-60 promyelocytic leukemia cells. Therefore cord blood and tumor derived exosomes provided a good source for adoptive immunotherapy.

Keywords: adoptive immunotherapy, cord blood, dendritic cells, cytotoxic T lymphocytes, exosome 


\section{Significance}

In this study, we demonstrate through detections of cytotoxicity and protein expression that the obtained CTLs were amplified to a specific direction and possessed high tumor killing activity in vitro. These UCB-CTLs generated by HL-60 derived exosomes activated DCs displayed specific cytotoxicity to HL-60 promyelocytic leukemia cells.

\section{Introduction}

Adoptive immunotherapy for tumor treatment is the infusion of immune cells with anti-tumor activity to directly kill the tumor cells or to trigger an immune response against the tumor cells. The common cell types infused include cytokine-induced killer (CIK) cells, natural killer cells (NK cells) and cytotoxic T lymphocytes (CTLs). ${ }^{[1-3]}$ CTLs are $\mathrm{CD}^{+} \mathrm{CD} 8^{+}$ T cells with MHC I restriction that can specifically and rapidly kill the target tumor cells. ${ }^{[4]}$ Although adoptive immunotherapy using autologous tumor antigen-specific $\mathrm{T}$ cells or $\mathrm{T}$ cells stimulated by tumor antigen in vitro has been shown to be effective for tumors, there are several complications. Cancer patients usually have immune hypofunction. ${ }^{[5]}$ Continuous chemotherapy and radiotherapy will harm immune cells making in vitro amplification difficult. Thus it is necessary to find alternative sources of immune cells that can work well.

Umbilical cord blood (UCB) is readily available that contains rich hematopoietic stem cells (HSCs) and immune cells. ${ }^{[6]}$ The UCB-derived HSCs possess the potential of multi-directional differentiation, and they can be differentiated and amplified in the presence of hematopoietic growth factor. ${ }^{[7]}$ At present, the UCB can be stored for a long time in a deep-freezing state. ${ }^{[8]}$ Through the induction of combinations of cytokines, large quantities of immune cells will be produced, including CIK cells and NK cells. ${ }^{[9]}$ However, there are only a few reports on the amplification of CTLs from umbilical cord blood.

Dendritic cells (DCs) are the most important antigen-presenting cells (APCs). ${ }^{[10]}$ DCs-based vaccinations have been demonstrated to be effective in inducing antigen-specific CTL responses. ${ }^{[11]}$ It is known that frozen thawed cells or weak acid washed cells are a simple method for obtaining cell associated antigens. ${ }^{[12]}$ Recently, researchers have found that the exosomes from tumor cells contains abundant tumor antigens and have many antigenic 
epitopes. ${ }^{[13]}$ MHC I molecules and HSP associated with antigen presentation, have stronger immunogenicity. ${ }^{[14]}$ In this study, we first cultured DC cells and CTL cells simultaneously using samples of cord blood and optimized the efficiency of in vitro amplification. Then, the tumor antigen information was delivered to the CTLs using tumor derived exosomes activated DC cells. The CTLs obtained were then detected for interferon (IFN)- $\gamma$ secretion and cytotoxicity. The main objectives of this work are to study whether DCs loaded with De miRNA exosome from tumor can induce antigen-specific CTL responses.

\section{Materials and methods}

\section{Blood sample}

Umbilical cord blood $(n=12)$ was collected from pregnant women with full-term pregnancy and vaginal delivery at Department of Gynaecology and Obstetrics, Qilu Hospital of Shandong University. Informed consent was obtained from the pregnant women, and the protocol was approved by Ethics Committee of Qilu Hospital of Shandong University. The samples were collected into blood bags containing sodium citrate anticoagulant and started the laboratory treatment within $24 \mathrm{~h}$.

\section{Isolation of exosomes from tumor cell lines HL-60}

The human promyelocytic leukemia cell line HL-60 was purchased from the American Type Culture Collection (Manassas, VA, USA) and cultured in RPMI-1640 medium (Roswell Park Memorial Institute-1640, Hyclone, Massachusetts, USA) supplemented with $10 \%$ fetal bovine serum (FBS, Ultro-protein Ji'nan, China) in an incubator at $37^{\circ} \mathrm{C}$ with $5 \% \mathrm{CO}_{2}$. For exosome collection, the cell density was adjusted to $4 \times 10^{6} / \mathrm{mL}$ with fresh complete medium containing 90\% RPMI-1640, 10\% FBS (centrifuged at 100,000 g for $6 \mathrm{~h}$ first to remove exosomes first). After $48 \mathrm{~h}$, the leukemia cell-conditioned medium was collected and centrifuged at $300 \mathrm{~g}$ to remove the floating cells and the supernatant were further filtered using a $0.22 \mu \mathrm{m}$ filter and eventually ultracentrifuged with $100,000 \mathrm{~g}$ for $10 \mathrm{~h}$ at $4^{\circ} \mathrm{C}$. The exosome pellets in the bottom were resuspended in $10 \mathrm{~mL}$ deionized water to broken the exosomes membrane. The supernatant were further filtered using10 Kd ultrafiltration at 4000 $\mathrm{g}$ for $30 \mathrm{~min}$ to remove microRNA and stored at $-80{ }^{\circ} \mathrm{C}$ for further use. The final 
quantification of exosomal proteins was measured by a BCA kit (Thermo, USA) according to the manufacturer's recommendations. We could collect $75 \mu \mathrm{g}$ exosome derived proteins from $150 \mathrm{~mL}$ supernatant.

The morphology of the exosomes was observed using transmission electron microscope (TEM, JEOL-1200EX, JEOL Ltd., Tokyo, Japan). Exosomes were identifed by specifc antibodies for CD63 (ab59479, dilution 1:3,000, Abcam), CD9 (ab2215, dilution 1:1,000, Abcam), heat shock protein 70 (Hsp70, \#4872, dilution 1:1,000, Cell signaling Tech.), Lamp 1 (ab24170, dilution 1:1,000, Abcam), and HLA (human leukocyte antigen)-I (ab126237, dilution 1:2,000, Abcam), with detection of beta-actin (ab8226, dilution 1:1,000, Abcam) used as a loading control for western blotting analysis.

\section{Preparing DCs from human umbilical cord blood}

DCs were prepared as previously described with certain modifications. ${ }^{[15]}$ Briefly, fresh UCB samples were diluted 1:2 in PBS. After centrifugation in Ficoll lymphocyte separation liquid (GE Healthcare Life Sciences, Shanghai, China) at $400 \mathrm{r} / \mathrm{min}$ for $35 \mathrm{~min}$, mononuclear cells (MNCs) were collected from the interface and washed twice in PBS by centrifugation at $200 \mathrm{r} / \mathrm{min}$ for $10 \mathrm{~min}$. MNCs were then cultured with RPMI-1640 medium supplemented with $10 \%$ FBS and the non-adherent cells were collected for subsequent CTL culture after $2 \mathrm{~h}$. Adherent cells were used to culture DCs with $20 \mathrm{ng} / \mathrm{mL}$ stem cell factor (SCF), $100 \mathrm{ng} / \mathrm{mL}$ granulocyte-macrophage colony-stimulating factor (GM-CSF), and $5 \mathrm{ng} / \mathrm{mL}$ Interleukin-4 (IL-4, all form Sigma, Missouri, USA). The culture medium was changed every 3 days and the exosome derived antigens were added to the DC culture at day 8 (50 $\mu \mathrm{g}$ exosome derived protein was added to $1 \times 10^{6} \mathrm{DC}$ cells). The morphology of DCs was examined under a phase contrast microscope and scanning electron microscope (SEM, Hitachi S-2400, Hitachi, Tokyo, Japan). Tumor necrosis factor alpha (TNF- $\alpha, 20 \mathrm{ng} / \mathrm{mL}, \mathrm{R} \& \mathrm{D}$, Minnesota, USA) was then added to these exosome derived antigens-loaded DCs (defined as HL-60-Ex-DCs group) to induce cell maturation at day 9. On the 10th day, the HL-60 exosome-primed DCs were collected as effector cells to mix with CTL cells. The workflow is shown in the Figure 1. To prepare negative controls 1 , DCs were cultured and matured in the presence of ultrasonic broken HL-60 cells membrane protein extract on day 8 with the same protein concentration 
(defined as HL-60mPr-DCs group), or without any antigen activation(defined as DCs group).

\section{Uptake of HL-60-Ex by DCs visualized with fluorescent labelling}

HL-60-Ex suspension was labelled with $\mathrm{DiO}$ [green] (3,3'- dioctadecyloxacarbocyanine, perchlorate, Biotium, Fremont, CA, USA) for $15 \mathrm{~min}$. Then HL-60-Ex were washed with PBS twice. The DCs were cultured on glass coverslips in a six-well plate and dyed in medium containing $5 \mu \mathrm{M}$ DID [red] (1,1'- dioctadecyl-3,3,3,3- tetramethylindodicarbocyanine, Biotium) at $37{ }^{\circ} \mathrm{C}$ for $20 \mathrm{~min}$ and then incubated with DiO-labelled HL-60-Ex $(50 \mu \mathrm{g}$, $50 \mu \mathrm{g} / \mathrm{mL}$ ) for $5 \mathrm{~h}$. After fixation with $4 \%$ paraformaldehyde for $20 \mathrm{~min}$, the cells were dyed with DAPI [blue] (4',6- diamidino-2- phenylindole, Sigma) for 2min. DCs were washed with PBS twice and observed with a fluorescence microscope (IX-71, Olympus, Tokyo, Japan).

\section{Culture scheme for umbilical cord blood-derived CTLS}

RPMI-1640 containing 10\% PBS was used as the basic medium. On Day 0, non-adherent cord blood mononuclear cells were cultured in basic medium supplemented with 50ng/mL Interferon-gamma (IFN- $\gamma$, R\&D, Minnesota, USA), 10ng/mL IL-15 (Millipore, MA, USA), with the cell density adjusted to $5 \times 10^{6} / \mathrm{mL}$. The medium was replaced on Day 1 , and the cells were cultured with $20 \mathrm{ng} / \mathrm{mL}$ SCF (Sigma) and FMS-like tyrosine kinase 3 ligand (FLT-3L, Millipore, MA, USA), 50 ng/mL anti-CD3, anti-CD28 (both from Beijing T\&L Biotechnology Co., Ltd. Beijing, China) and 500 U/mL IL-2 (Shanghai Huaxin High Biotechnology Co., Ltd. Shanghai, China). The medium was then replaced on Day 4, and the medium contained $20 \mathrm{ng} / \mathrm{mL}$ SCF and FLT-3L, $10 \mathrm{ng} / \mathrm{mL}$ IL-15 and $500 \mathrm{U} / \mathrm{mL}$ IL-2 from Day 4 to Day 7. The medium was replaced on Day 8, and $10 \mathrm{ng} / \mathrm{mL}$ IL-15 and $500 \mathrm{U} / \mathrm{mL}$ IL-2 was added from Day 8-Day 15. The cells were cultured in a humidified incubator at $37^{\circ} \mathrm{C}$ with $5 \% \mathrm{CO}_{2}$. The medium was added or replaced depending on cell growth throughout the experiment so that the cell density was maintained at $5 \times 10^{6} / \mathrm{mL}$. At day 12 , lymphocytes were mixed with DC at the ratio of 100: 1 (CTL:DC) and cultured for further three days. The cells were then harvested at day 15. The workflow is shown in the Figure 1.

\section{Immunophenotypic identification to DCs and CTL cells}

The following mAbs were used to characterize DCs and CTLs surface phenotypes: 
phycoerythrin (PE)-conjugated anti-CD80; PE-anti-CD83; PE-anti-CD86, PE-anti-HLA-DR; PE-anti-CD3 and fluorescein isothiocyanate (FITC)-anti-CD8 monoclonal antibodies were used (all from (BD PharMingen, San Diego, CA).The harvested cells were washed, resuspended in PBS supplemented with $1 \%$ BSA and $0.01 \% \mathrm{NaN}_{3}$, and incubated with the mAbs for 30 minutes on ice. The cells were washed twice in PBS and $1 \times 10^{4}$ of those labeled cells were subjected by Guava easyCyte 6HT (Millipore, USA). Results were analysed by guavaSoft 3.1.1 (Millipore, USA).

\section{Analysis of IFN- $\gamma$ and TNF- $\alpha$ secretion from CTLs using enzyme-linked immunosorbent assay (ELISA)}

The culture medium of CTL cells was transferred to a centrifuge tube and centrifuged at $1,500 \times g$ for $10 \mathrm{~min}$ at $4{ }^{\circ} \mathrm{C}$ to collect supernatant at day 15 . The secretion of IFN- $\gamma$ and TNF- $\alpha$ from each group of CTLs was determined by ELISA according to the manufacturer's instruction $(R \& D)$. Assays were performed in triplicate.

\section{Cytotoxicity of the CTLs}

To determine the specificity of CTL, the human leukemia B-cell line BALL-1 (American Type Culture Collection) was selected as negative control. HL-60 and BALL-1 in logarithmic growth phase were taken as target cells, with cell density adjusted to $1 \times 10^{5} / \mathrm{mL}$. The cells harvested after culture for 15 days were the experimental cells. Depending on the effector/target ratio, CTL density was adjusted to $1 \times 10^{6} / \mathrm{m} 1,2 \times 10^{6} / \mathrm{m} 1$ and $3 \times 10^{6} / \mathrm{mL}$, respectively. Then CTLs and target cells (each $100 \mu \mathrm{l}$ ) were mixed together at the effector/target ratio of 10:1, 20:1 and 30:1, respectively. Control wells were set for target cells and CTLs, respectively, along with the blank control wells. The final volume was 200ul/well, and each group had 8 replicates. The cells were cultured at $37^{\circ} \mathrm{C}$ in a $5 \% \mathrm{CO}_{2}$ incubator. One row of wells was selected at $3 \mathrm{~h}$ and $6 \mathrm{~h}$, respectively, and 10ul CCK-8 was added and mixed well. Then the cells were further cultured for 1h, and OD of each well was detected at 450nm. Cell cytotoxicity was calculated as follows: the average of the 3 replicates was taken, and the cytotoxicity of effector cells was calculated and expressed as killing rate 
(\%): cytotoxicity=1- (OD value of target and effector mixture -OD value of effector cells)/OD value of target cells $\times 100 \%$.

\section{Statistical analysis}

All data were expressed as mean \pm standard deviation (mean \pm s). Statistical analyses were performed using SPSS 12.0 software. Paired $t$-test was adopted to compare the differences between the two samples, and $P<0.05$ indicated significant difference.

\section{Results}

\section{Generation of DCs}

Adherent cells from cord blood MNCs were round and regular with a diameter of approximately $8 \mu \mathrm{m}$. On day 3, cells became larger and their shape also started to become irregular with burr like protuberances. During the late period of culture, DCs shed from the bottom into the medium (Figure. 2A). After 9 days of induction, the expression of CD80, CD83, CD86 and HLA-DR was gradually increased in DCs stimulated with GM-CSF, IL-4, and TNF- $\alpha$. Figure 2B shows the results from one of the cord blood samples analyzed. On day 3, approximately $(21.34 \pm 1.97) \%,(16.3 \pm 1.32) \%,(7.9 \pm 0.88) \%$, and $(12.6 \pm 1.17) \%$ of DCs expressed CD80, CD83, CD80, and HLA-DR, respectively and these numbers increased to $(59.2 \pm 3.48) \%,(66.34 \pm 5.36) \%,(75.41 \pm 6.85) \%$ and $(67.93 \pm 5.11) \%$ on day 9 (Fig. 2C). These results indicate that the monocytes isolated were successfully transformed into mature DCs. But the results show that the addition of exosome or membrane protein extract does not have much effect on the maturity of DCs (data not shown).

\section{Typical characteristics of HL-60-Ex and effective uptake of HL-60-Ex by DC}

To further obtain HL-60-Ex, we used gradient ultracentrifugation to extract exosome from the culture medium. The obtained exosomes were yellow at the bottom of the test tube (Fig. 3A). The exosomes had an average diameter of 50-200 nm in TEM images (Fig. 3B). Western blotting analysis indicated that HL-60-Ex expressed CD63, CD9, Hsp70 and HLA-I proteins, but did not express the lysosome marker Lamp 1 (Fig. 3C). Hence, HL-60-Ex expressed the typical markers of exosome and was used in the following experiments. 
Under fluorescence microscopy, we observed that HL-60-Ex, DC cell membrane and DC cell nucleus labeled with DIO, DID and DAPI show green, red and blue fluorescence, respectively. After merging the images, we found that HL-60-Ex (green) had been adsorbed or engulfed by DC cells (red), indicating that HL-60-Ex successfully entered the DC cells(Figure 4D-G).

\section{Proliferation of CTLs and immunophenotype analysis}

After culture for 15 days, the cell number increased considerably, and clone spheres formed by fast cell proliferation were observed under the microscope (Figure 4A). The proliferation rate of CTL was significantly accelerated after DC stimulation, whereas HL-60mPr-DCs-CTL group and HL-60-Ex-DCs-CTL group was faster than that in DCs-CTL group $(P<0.05)$. Direct contact between DC and CTL can be seen under SEM (Figure 4B). In the most amplified HL-60-Ex-DCs-CTL group, the average cell count increased from $4.6 \times 10^{7}$ before amplification to $1.21 \times 10^{9}$ after 15 days amplification, and the average proliferation rate was $(2630 \pm 283) \%$ (Figure 4C). As shown by flow cytometry analysis, the proportion of $\mathrm{CD}^{+} \mathrm{CD}^{+}$cells in the HL-60-Ex-DCs-CTL group was as high as $(82.05 \pm 3.7) \%$ after 15 days in vitro culture (Figure 4D, E).

\section{IFN- $\gamma$ and TNF- $\alpha$ secretion in $C T L$}

Secreted cytokines IFN- $\gamma$ and TNF- $\alpha$ are important for CTL cells in execution of cytotoxic function. According to the results of ELISA, the concentration of IFN- $\gamma$ in

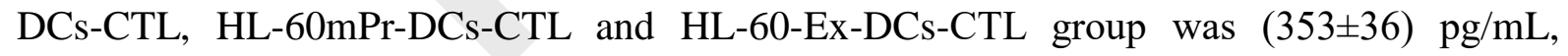
$(408 \pm 45) \mathrm{pg} / \mathrm{mL}$ and $(418 \pm 39) \mathrm{pg} / \mathrm{mL}$, respectively. There was no statistical difference between the three groups. The average concentration of TNF- $\alpha$ is $(138 \pm 9) \mathrm{pg} / \mathrm{mL},(294 \pm 21)$ $\mathrm{pg} / \mathrm{mL}$ and $(284 \pm 17) \mathrm{pg} / \mathrm{mL}$ in supernatant of three groups, respectively, whereas the values of the DCs-CTL group were significantly lower than those of the other two groups $(P<0.05$, Figure 5A).

\section{Cytotoxicity of the generated HL-60 specific CTLs.}

The killing effect of HL-60 specific CTL on tumor cells induced by sensitized DCs was compared under different effector/target ratio and shown in Figure 5B. It was found that 
CTLs sensitized by HL-60 exosome showed a better killing activity on HL-60 cells compared with control group. The higher the effector/target ratio, the better the killing effect. There was no statistical difference between the membrane protein extract or exosome induced DC-CTL on cytotoxicity to HL-60 cells $(P>0.05)$. But both of HL-60mPr-DCs-CTL and HL-60-Ex-DCs-CTL groups has far greater killing rate on HL-60 cells than the BALL-1 cells $(P<0.01)$. These results suggested that a specific cytotoxicity of HL-60-DC-activated T-lymphocytes against HL-60 tumor cells.

\section{Discussion}

DCs based immunotherapy, including CTLs, are the dominant effectors in immunotherapy due to its potentially superior efficacy and specificity. ${ }^{[3]}$ Several approaches involving the use of whole tumor RNA, tumor lysates, apoptotic or necrotic debris and fusion have been used to activate DCs. ${ }^{[16]}$ The present study aimed to generate HL-60 specific CTLs in vitro through exosomes stimulation to DCs. Exosomes are released in vitro by many types of cells including tumor cell lines and antigen presenting cells. ${ }^{[16,17,18]}$ Our results revealed that $>82.05 \%$ of the cells generated from the cord blood samples were $\mathrm{CD} 3^{+} \mathrm{CD} 8^{+}$.

The key point of tumor immunotherapy is to select the appropriate tumor antigen. The commonly used tumor antigens include tumor cells freeze-thaw extract, irradiated tumor cells, apoptotic body, heat shock protein, and acid eluted peptide. ${ }^{[12,19,20]}$ But the complexity of these components may lead to autoimmune diseases. Exosomes are small membrane vesicles secreted into the extracellular compartment by exocytosis. These subcellular membrane vesicles from endosomal origin are secreted upon fusion of multi-vesicular bodies with the plasma membrane ${ }^{[13]}$. As a consequence, exosomes have a "cellular" membrane oriented with a limited variety of proteins derived from the cytosol, endocytic compartment membranes, and plasma membranes ${ }^{[21]}$. Tumor derived-exosomes are immunogenic and exosomes may be a novel source of tumor-specific antigens which may be used for T-cell cross priming and be relevant for immuno-interventions. ${ }^{[14]}$ Exosomes contains MHC I molecules and HSP, which has a strong immunogenicity and relatively simple composition. ${ }^{[22,23]}$ A trace of HSP peptide complexes is enough to trigger strong CTL reaction 
because APCs express HSP receptors on its surface. ${ }^{[24]}$ Exosomes derived from tumor cell lines transfer shared tumor antigens to DCs and, thus stimulate T cells in an MHC class I dependent way, and provide cross-protection against syngeneic and allogeneic tumors in mice ${ }^{[25]}$. Although noncoding RNA could be considered as a potential diagnostic biomarker, but it also affects the function of DCs. ${ }^{[26]}$ So our technology removed the miRNA in exosome, thus avoiding the immune escape caused by miRNA. ${ }^{[27]}$

Our results showed that DCs can be obtained from UCB and express costimulatory molecules using SCF, GM-CSF and IL-4 combination. FACS analyses revealed that the expression of CD80, CD83, CD86 and HLA-DR was gradually increased in the DCs after induction. The primary function of HLA-DR is to present antigens to the immune system for eliciting T cells responses. ${ }^{[28]}$ Therefore, the increase of HLA-DR expression is beneficial for DCs to stimulate T cells. CD80 and CD86 are the important costimulatory molecules on DCs. These two markers engage in the interaction between APCs and T cells. CD83 is the typical mature marker of DCs. ${ }^{[29]}$ Although autologous CTLs are preferred, healthy individuals with the same HLA antigen or partially matched HLA antigen are also a source of specific CTLs. ${ }^{[30,31]}$ The duration of CTLs culture is usually short and CTLs can proliferate only to a limited extent. The proliferation of patient autologous CTLs is made more difficult by the state of immune hypofunction and continuous chemotherapy and radiotherapy. HSCs derived from UCB can be used as an alternative. Although the killing activity of specific CTLs derived from the UCB is lower than that of CTLs derived from adult peripheral blood, the concentrations of T-cell precursors and helper T-cell precursors are comparable. ${ }^{[32,33]}$ These CTLs can proliferate intensively under the stimulation from heterogenic antigen and they are still qualified for adoptive immunotherapy. ${ }^{[34]}$ Our cell culture scheme was modeled on the experiment scheme for adult CTLs. Anti-CD3, anti-CD28 and IL-2 were added to induce intensive proliferation and differentiation into tumor-specific CTLs with high tumor killing activity. ${ }^{[35]}$ Anti-CD28 can induce the activation of $\mathrm{CD} 4^{+} \mathrm{T}$ cells and $\mathrm{CD}^{+} \mathrm{T}$ cells simultaneously. ${ }^{[36]}$ IL-2 and IL-15 are believed to perform similar functions, both of which stimulate the proliferation of T cells, induce the production of CTLs. ${ }^{[37]}$ FLT-3L is a cytokine that promotes the formation and differentiation of HSCs, several hematopoietic cells and their 
precursors. ${ }^{[38]}$ Studies have shown that FLT-3L promotes the early growth of T cells. ${ }^{[39]}$ SCF can induce the proliferation of HSCs. In a short-term culture using G-CSF and SCF, the number of CFUs was increased by 6-8 times within about one week. ${ }^{[40]}$ In the present study, CTLs were cultured with IFN- $\gamma$, IL-15, SCF, FLT-3L, anti-CD3, anti-CD28 and IL-2. The CTLs proliferated intensively and displayed a strong tumor killing activity.

CTLs can kill the target cells by exocytosis of cytoplasmic particles and release of particle inclusions. ${ }^{[41]}$ The released particles contain perforin and granzyme. ${ }^{[42]}$ CTLs can also secrete proinflammatory cytokines, including TNF- $\alpha$ and IFN- $\gamma$, which are regulatory of the immune function. ${ }^{[43]}$ Our ELISA results showed that the expressions of TNF- $\alpha$ and IFN- $\gamma$ in HL-60-Ex-DCs or HL-60mPr-DCs activated CTLs were upregulated compared with the control group, whereas the TNF- $\alpha$ were the most significantly upregulated. The cytotoxicity assays further revealed that HL-60-Ex-DCs-activated T-lymphocytes were remarkably more cytotoxic than HL-60mPr-DCs-activated T-lymphocytes, or simply DCs-activated T-lymphocytes. Our experiments also indicated that these HL-60-Ex-DCs or HL-60mPr-DCs activated CTLs exerted little influence on unrelated Ball- 1 cells. These $\mathrm{CD}^{+} \mathrm{CD}^{+} \mathrm{T}$ cells generated by HL-60-Ex-DCs displayed specific cytotoxicity towards HL-60.

In summary, we demonstrate through detections of cytotoxicity and protein expressions that the obtained CTLs were amplified towards a specific direction and possessed high tumor killing activity in vitro. These UCB-CTLs generated by HL-60 derived exosomes activated DCs displayed specific cytotoxicity towards HL-60 promyelocytic leukemia cells.

\section{Funding}

This work was supported by Shandong Province Science Fund (2017GSF18155, 2017GSF218015, ZR2018MH012), Quancheng Industrial Leadership Talents Support Plan (2017018), and Jinan Medical \& Health Science and Technology Innovation Plan (201704066).

\section{Data Availability Statement}

The datasets generated during and/or analysed during the current study are available from the corresponding author on reasonable request. 


\section{References}

1. Yu R, Yang B, Chi X, et al. Efficacy of cytokine-induced killer cell infusion as an adjuvant immunotherapy for hepatocellular carcinoma: a systematic review and meta-analysis. Drug Des. Devel. Ther. 2017; 11:851-864.

2. Lazear E, Ghasemi R, Hein SM, et al. Targeting of IL-2 to cytotoxic lymphocytes as an improved method of cytokine-driven immunotherapy. Oncoimmunology. 2017; 6:e1265721.

3. Goto T, Nishida T, Takagi E, et al. Programmed death-ligand 1 on antigen-presenting cells facilitates the induction of antigen-specific cytotoxic $\mathrm{T}$ lymphocytes: application to adoptive T-cell immunotherapy. J. Immunother. 2016; 39:306-315.

4. Muraro M, Mereuta OM, Saglio F, et al. Tumor-associated-antigens or osteosarcoma cell line lysates: two efficient methods for in vitro generation of CTLs with special regard to MHC-I restriction. Cell Immunol. 2011; 266:123-129.

5. Shionoya Y, Kanaseki T, Miyamoto S, et al. Loss of tapasin in human lung and colon cancer cells and escape from tumor-associated antigen-specific CTL recognition. Oncoimmunology. 2017; 6:e1274476.

6. Ballen KK, Gluckman E, Broxmeyer HE. Umbilical cord blood transplantation: the first 25 years and beyond. Blood. 2013; 122:491-498.

7. Sotnezova EV, Andreeva ER, Grigoriev AI, et al. Ex vivo expansion of hematopoietic stem and progenitor cells from umbilical cord blood. Acta Naturae. 2016; 8:6-16.

8. Broxmeyer HE, Lee MR, Hangoc G, et al. Hematopoietic stem/progenitor cells, generation of induced pluripotent stem cells, and isolation of endothelial progenitors from 21-to 23.5-year cryopreserved cord blood. Blood. 2011; 117: 4773-4777.

9. Li Y, Schmidt-Wolf IG, Wu YF, et al. Optimized protocols for generation of cord blood-derived cytokine-induced killer/natural killer cells. Anticancer Res. 2010; 30: 3493-3499. 
10. Banchereau J, Briere F, Caux C, et al. Immunobiology of dendritic cells. Annu. Rev. Immunol. 2000;18:767-811.

11. Velten FW, Rambow F, Metharom P, et al. Enhanced T-cell activation and T-cell-dependent IL-2 production by CD83+, CD25high, CD43high human monocyte-derived dendritic cells. Mol Immunol. 2007;44:1544-1550.

12. Friedl J , Stift A, Paolini P, et al. Tumor antigen pulsed dendritic cells enhance the cytolytic activity of tumor infiltrating lymphocytes in human hepatocellular cancer. Cancer Biother Radiopharm. 2000; 15: 477-486.

13. Denzer K, Kleijmeer MJ, Heijnen HF, et al. Exosome: from internal vesicle of the multivesicular body to intercellular signaling device. J. Cell. Sci. 2000; 113:3365-3374.

14. André F, Schartz NE, Chaput N, et al. Tumor-derived exosomes: a new source of tumor rejection antigens.Vaccine. 2002; 20(Supp14):A28-A31.

15. Lambert LA, Gibson GR, Maloney M, et al. Equipotent generation of protective antitumor immunity by various methods of dendritic cell loading with whole cell tumor antigens. J Immunother (1991). 2001; 24:232-236.

16. Wolfers J, Lozier A, Raposo G, et al. Tumor-derived exosomes are a source of shared tumor rejection antigens for CTL cross-priming. Nat. Med. 2001; 7:297-303.

17. Raposo G, Nijman HW, Stoorvogel W, et al. B lymphocytes secrete antigen-presenting vesicles. J. Exp. Med. 1996; 183:1161-1172.

18. Zitvogel L, Regnault A, Lozier A, et al. Eradication of established murine tumors using a novel cell-free vaccine: dendritic cell-derived exosomes. Nat. Med. 1998; 4:594-600.

19. Janetzki S, Palla D, Rosenhauer V, et al. Immunization of cancer patients with autologous cancer-derived heat shock protein gp96 preparations: a pilot study.Int. J. Cancer. 2000; $88: 232-238$.

20. Santin AD, Bellone S, Ravaggi A, et al. Induction of ovarian tumor specific CD8+ cytotoxic T lymphocytes by acid eluted peptide pulsed autologous dendritic cells. Obstet 
Gynecol. 2000; 96: 422-430.

21. Théry C, Zitvogel L, Amigorena S. Exosomes: composition, biogenesis and function. Nat. Rev. Immunol. 2002; 2:569-579.

22. Li QL, Bu N, Yu YC, et al. Ex vivo experiments of human ovarian cancer ascites-derived exosomes presented by dendritic cells derived from umbilical cord blood for immunotherapy treatment. Clin. Med. Oncol. 2008; 2:461-467.

23. Théry C, Boussac M, Véron P, et al. Protemic analysis of dendritic cell-derived exosomes: a secreted subcellular compartment distinct from apoptotic vesicles. J Immunol. 2001; 166:7309-7318.

24. Lipsker D, Ziylan U, Spehner D, et al. Heat shock proteins 70 and 60 share common receptors which are expressed on human monocyte-derived but not epidermal dendritic cells. Eur. J. Immunol. 2002; 32:322-332.

25. Srivastava PK. Purifi cation of heat shock protein-peptide complexes for use in vaccination against cancers and intracellular pathogens. Methods. 1997; 12:165-171.

26. Ma H, Xu Y, Zhang R, et al. Differential expression study of circular RNAs in exosomes from serum and urine in patients with idiopathic membranous nephropathy. Arch Med Sci. $2019 ; 15: 738-753$.

27. Palma J, Yaddanapudi SC, Pigati L, et al. MicroRNAs are exported from malignant cells in customized particles. Nucleic Acids Res. 2012; 40: 9125-9138.

28. Bottazzo G, Hanafusa T, Pujol-Borrell R, et al. Role of aberrant HLA-DR expression and antigen presentation in induction of endocrine autoimmunity. The Lancet. 1983; 2: 1115-1119.

29. Su H, Luo Q, Xie H, et al. Therapeutic antitumor efficacy of tumor-derived autophagosome (DRibble) vaccine on head and neck cancer. International Journal of Nanomedicine. 2015; 10: 1921-1930.

30. Møller J, Dickmeiss E, Jacobsen N, et al. Cytotoxic T cell activity after HLA-mismatch 
bone marrow transplantation. Tokai. J. Exp. Clin. Med. 1996; 21: 19-23.

31. Lucivero G, Dalla Mora L, Bresciano E, et al. Functional characteristics of cord blood T lymphocytes after lectin and anti CD3 stimulation. Differences in the way T cells express activation molecules and proliferate. Int. J. Clin. Lab. Res. 1996; 26: 255-261.

32. Risdon G, Gaddy J, Stehman FB. Broxmeyer HE. Proliferative and cytotoxic responses of human cord blood T lymphocytes following allogeneic stimulation. Cell Immunol. 1994; 154: 14-24.

33. Yee GC. The future of cell therapy. Pharmacotherapy. 1996; 16:109.

34. Gardiner CM, Meara AO. Reen DJ. Differential cytotoxicity of cord blood and bone marrow-derived natural killer cells. Blood. 1998; 91: 207-213.

35. Huang H, Yu H, Xiong SD, et al. Anti-tumor effects of mouse CTL of non-specific expansion. Chinese Journal of Microbiology and Immunology. 2001; 21:143-145.

36. Rosenberg SA, Yang JC, Sherry RM, et al. Durable complete responses in heavily pretreated patients with metastatic melanoma using T-cell transfer iinmunotherapy. Clin. Cancer. Res. 2011; 17: 4550-4557.

37. Waldmann TA, Dubois S. Tagaya Y. Contrasting roles of IL-2 and IL-15 in the life and death of lymphocytes:implications for immunotherapy. Immunity. 2001; 14: 105-110.

38. Hunte BE, Hudak S, Campbell D, et al. Flk2/flt3 ligand is a potent cofactor for the growth of primitive B cell progenitors. J Immunol. 1996; 156: 489-496.

39. Moore TA, Zlotnik A. Differential effects of Flk2/flt3 ligend and stem cell factor on murine thymic progenitor cells. J Immunol. 1997; 158: 4187-4192.

40. Moore MA. Umbilical cord blood: an expandable resource. J. Clin. Invest. 2000; 105:855-856.

41. Gross G, Wales T, Eshhar Z. Expression of immunoglobulin-T-cell receptor chimeric molecules as functional receptors with anti-body-type specificity. Proc Natl Acad Sci USA. 1989; 86: 10024-10028. 
42. Pule MA, Savoldo B, Myers GD, et al. Virus-specific T cells engineered to coexpress tumor-specific receptors: persistence and anti-tumor activity in individuals with neuroblastoma. Nat. Med. 2008; 14: 1264-1270.

43. Stastny MJ, Brown CE, Ruel C, et al. Medulloblastomas expressing IL13Ralpha2 are targets for IL13-zetakine+ cytolytic T cells. J. Pediatr. Hematol. Oncol. 2007; 29: 669-677.

Figure 1. Culture scheme for umbilical cord blood-derived DCs and CTLs.

Figure 2. Morphological observation and phenotypic analysis of DCs. A: Morphological observation of DCs from day 0 to day 9 ( $\times 200$ magnifcation); B\&C: Flow cytometric analysis of phenotypic changes in DCs upon in-vitro stimulations with GM-CSF, IL-4 and TNF- $\alpha$ on day 3 and day 12. $(\mathrm{n}=12$. $* P<0.05)$. B shows the results from one of the cord blood samples analyzed.

Figure 3. Typical characteristics of HL-60-Ex and uptake of HL-60-Ex by DC. A: The exosomes precipitated in the bottom of the tube. B: TEM showed the morphology of exosomes, which were 50-200 $\mathrm{nm}$ in diameter. C: Western blotting analysis indicated that HL-60-Ex expressed CD63, CD9, Hsp70 and HLA-I proteins. However, Lamp1 was not expressed. D-G: HL-60-Ex (DiO/green) are adsorbed on the surface of DC (DID/red) or engulfed in the DC after image overlay ( $\times 400$ magnifcation).

Figure 4. Morphological observation and phenotypic analysis of CTLs. A: Morphological observation of CTL from day 0 to day 15. (×200 magnifcation); B: Direct contact between DC and CTL can be seen under SEM (Bar=5 $\mu \mathrm{m})$; C: Growth curve of CTLs (CCK-8 assay);D\&E: Percentage of $\mathrm{CD}^{+} \mathrm{CD}^{+}$cell after amplification. $\left(\mathrm{n}=12{ }^{*} P<0.05\right)$. C shows the results from one of the cord blood samples analyzed.

Figure 5. Analysis of CTLs cell function. A: Concentrations of IFN- $\gamma$ and TNF- $\alpha$ from DCs-activated CTLs supernatant at day 15; B: Results of lymphocyte killing experiment of expanded CTLs (CCK-8 assay). (n=3. * $P<0.05)$ 


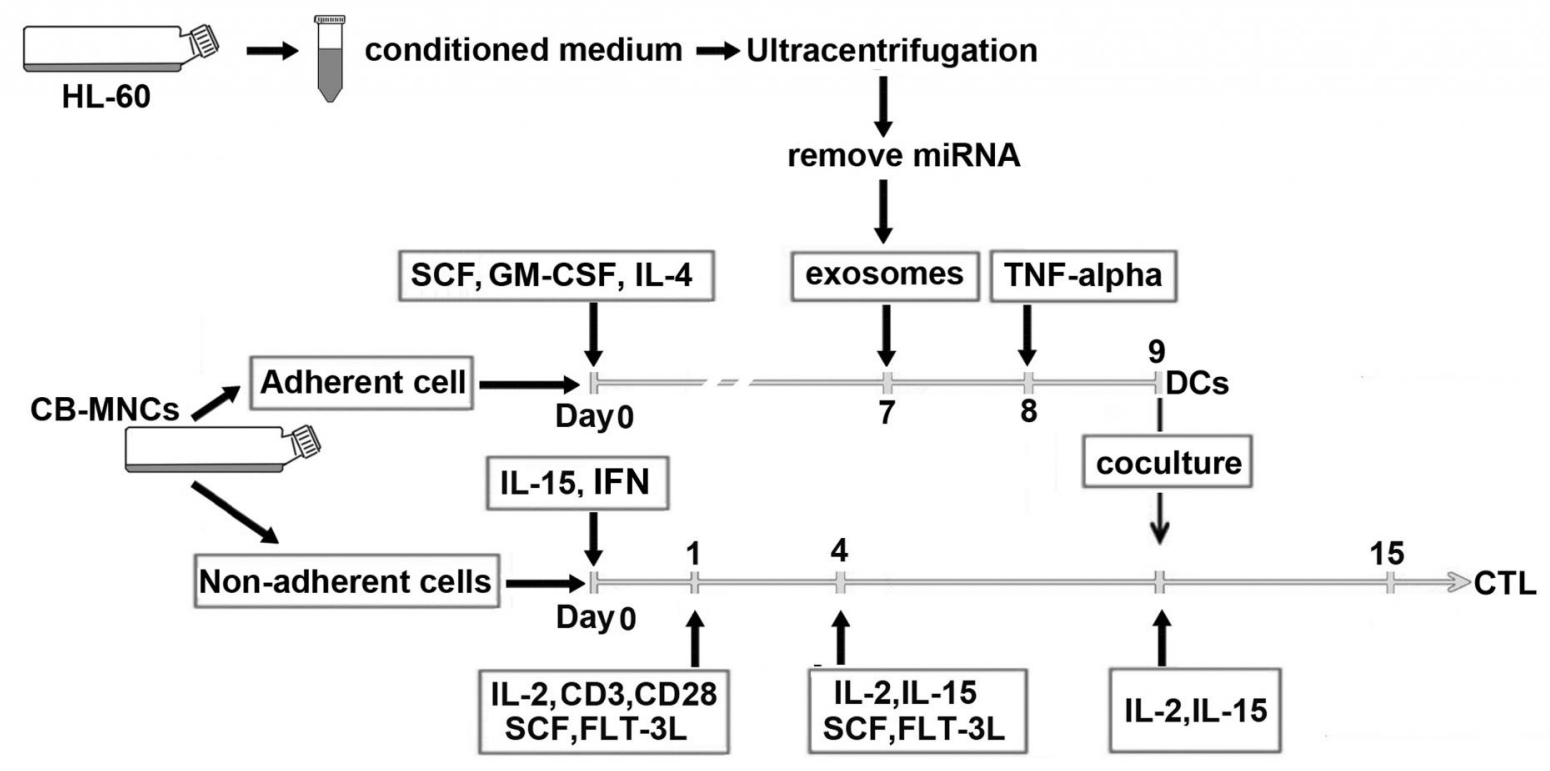


A
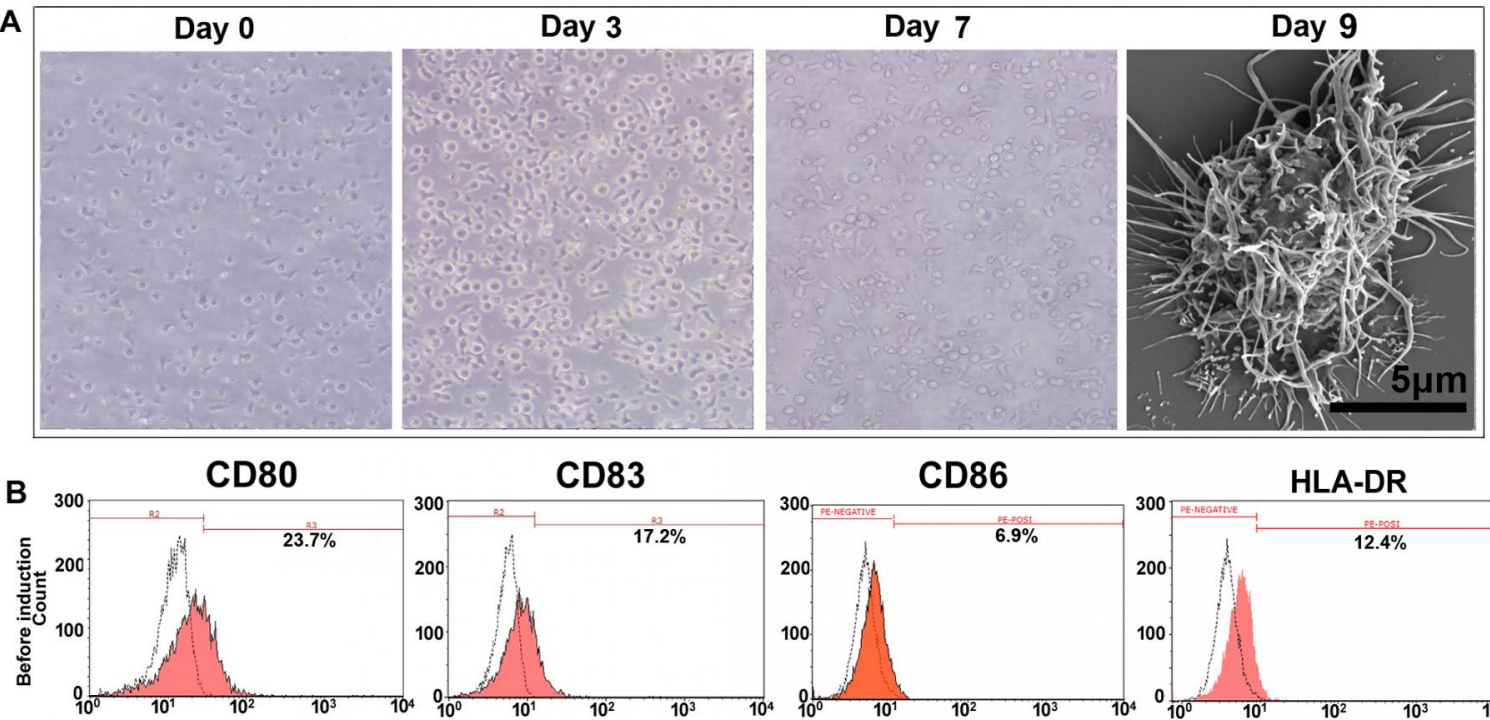

HLA-DR
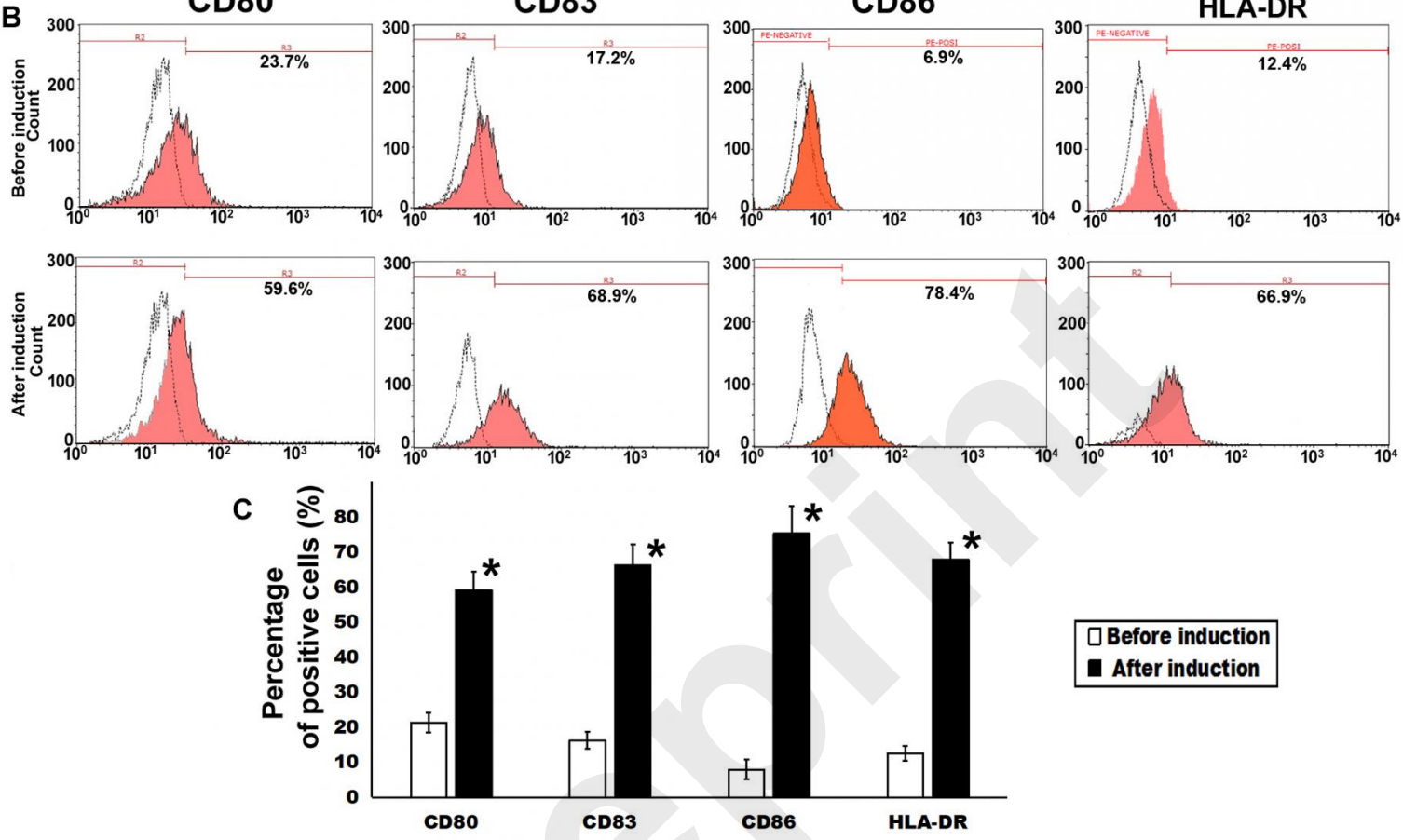

QBefore induction

- After induction 

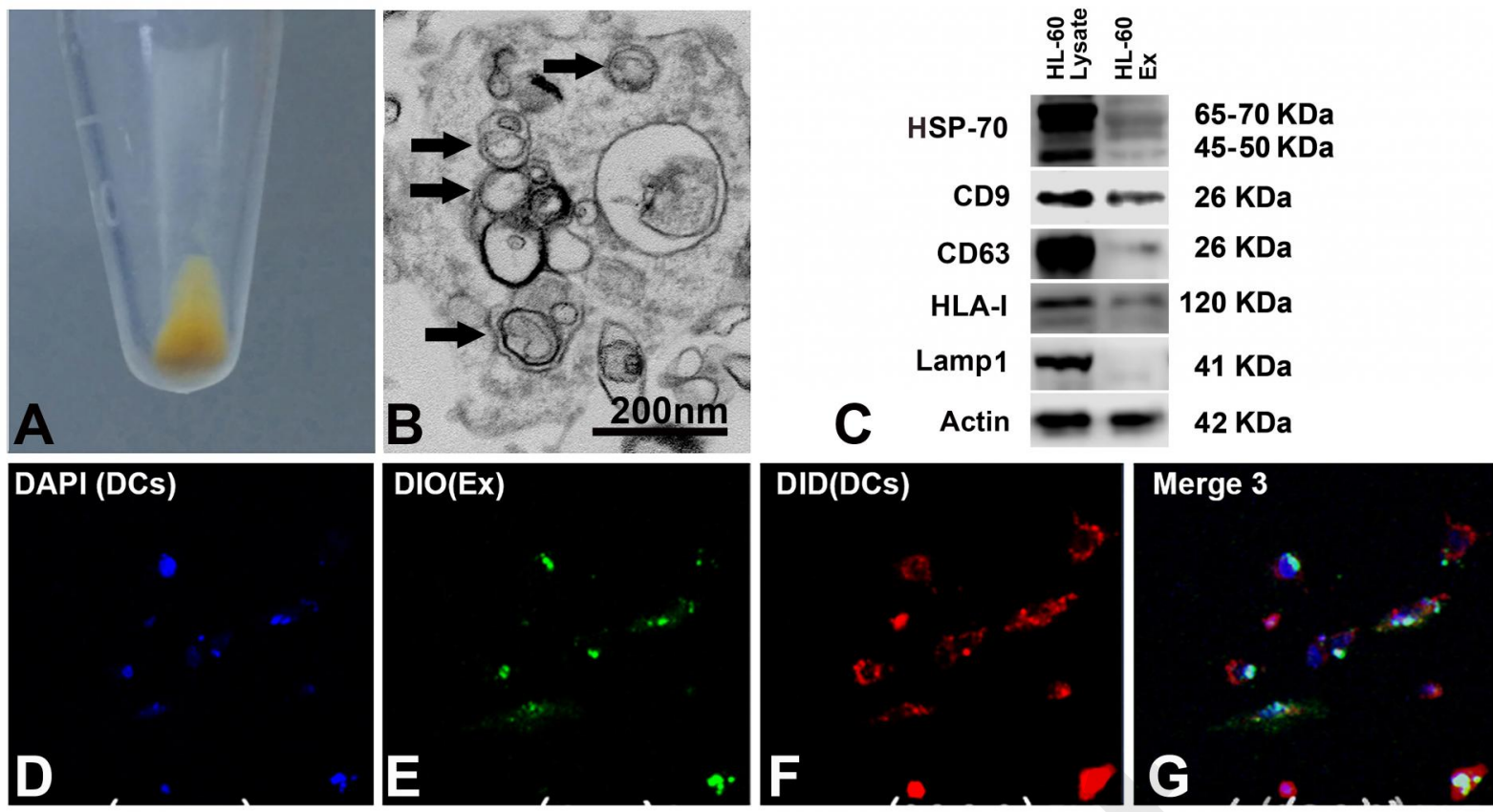

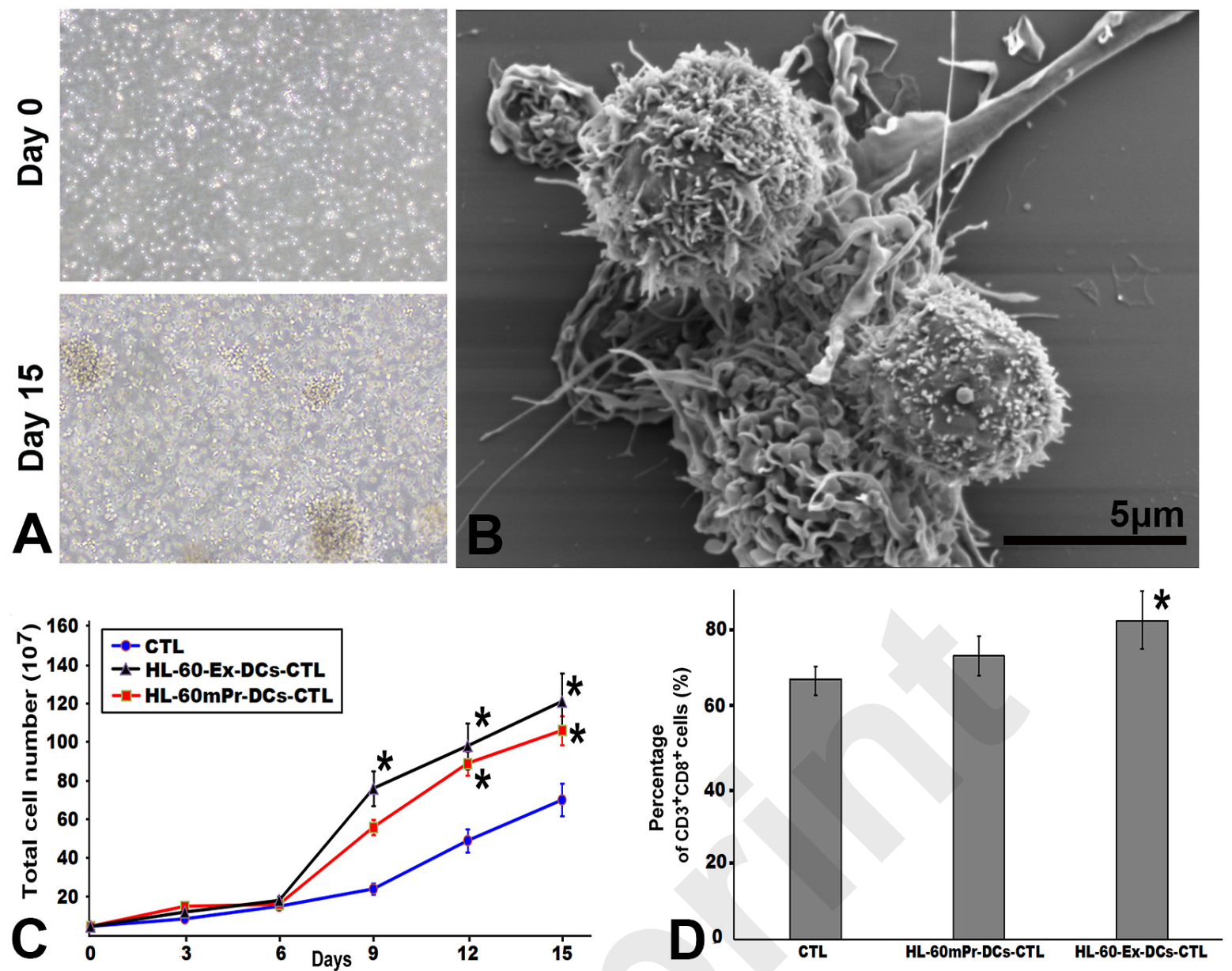

CTL
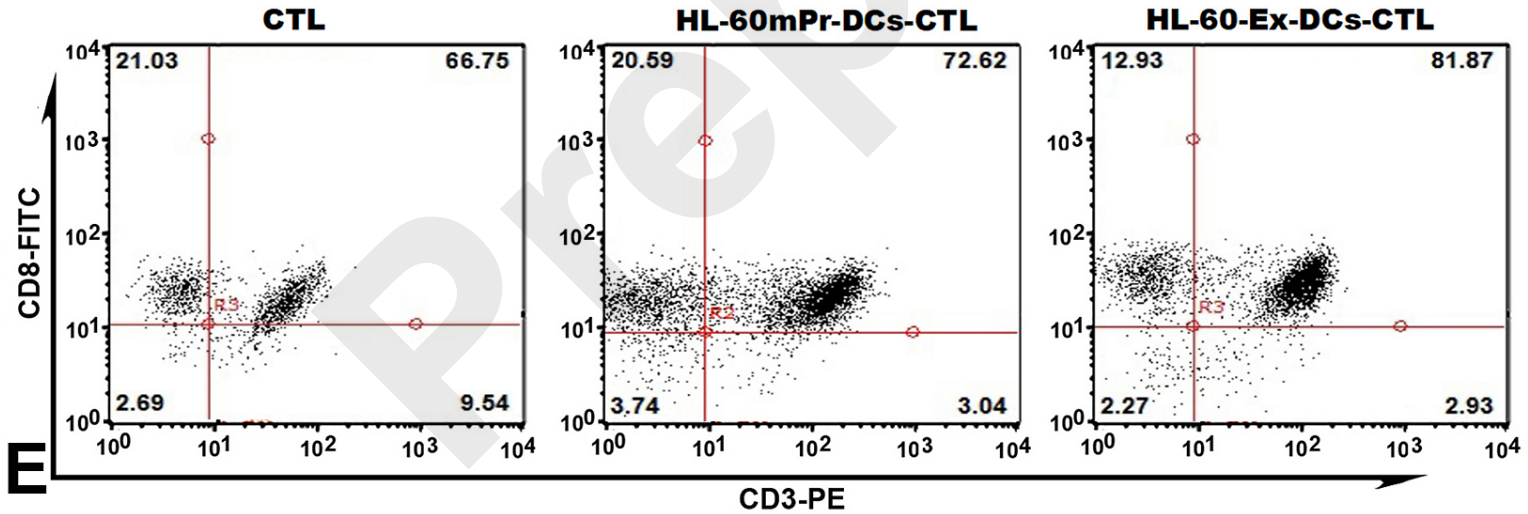

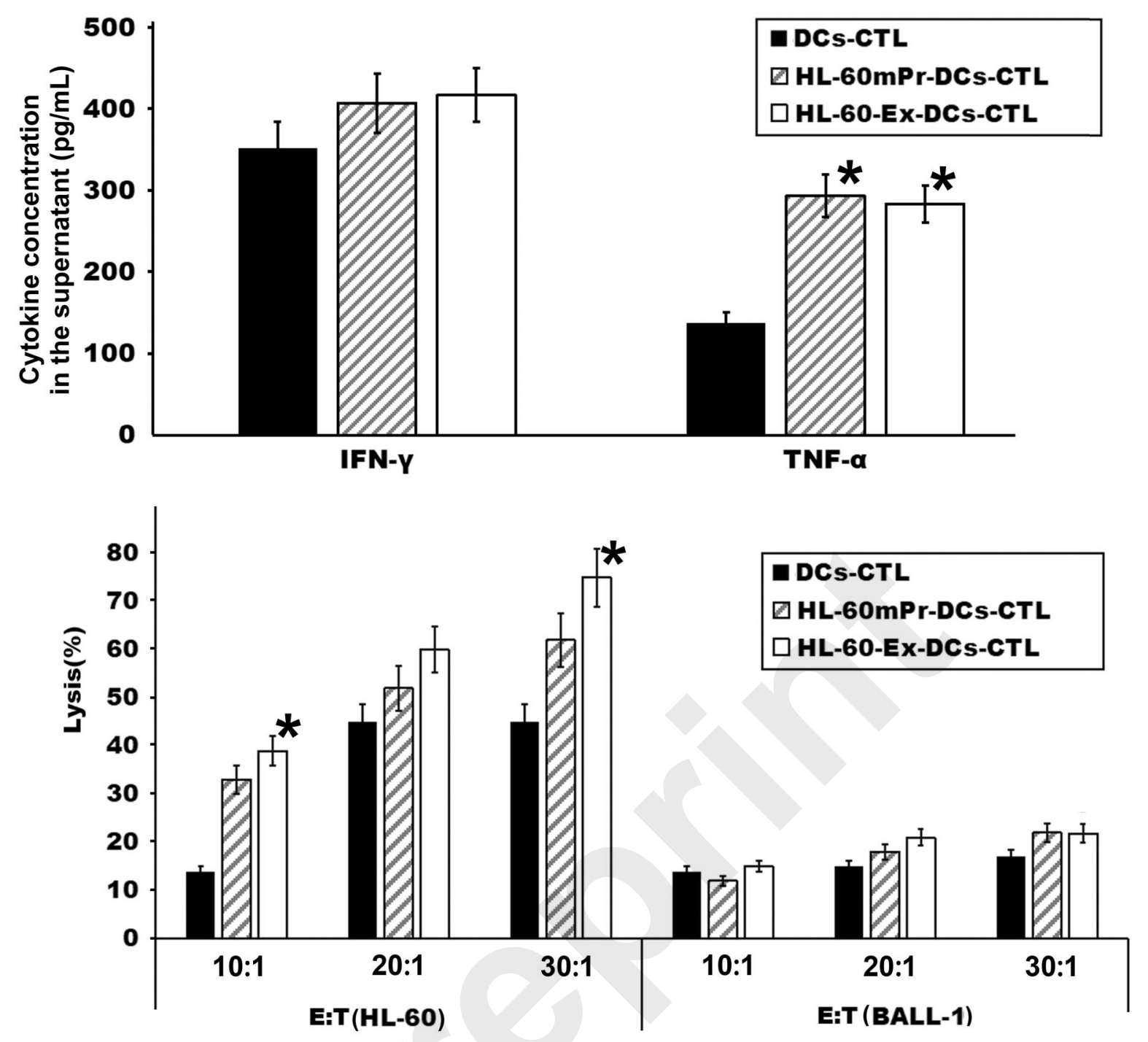